\title{
Reconfigurable Machine Tools
}

\author{
R.G. Landers ${ }^{1}$, B.-K. Min ${ }^{2}$, and Y. Koren ${ }^{2}$ (1) \\ ${ }^{1}$ University of Missouri - Rolla, Department of Mechanical Engineering, Rolla, Missouri \\ ${ }^{2}$ University of Michigan, Department of Mechanical Engineering, Ann Arbor, Michigan
}

\begin{abstract}
The uniqueness of Reconfigurable Manufacturing Systems (RMSs) is that the structure of the system as well as of its machines and controls can be rapidly changed in response to market changes (demand and products). A major component of RMSs is the reconfigurable machine tool (RMT). By contrast to conventional CNCs that are general-purpose machines, RMTs are designed for a specific, customized range of operation requirements and may be cost-effectively converted when the requirements change. In this paper, systematic design tools that have been recently developed for RMTs are reviewed, and three examples are provided to compare RMTs to traditional types of machine tools.
\end{abstract}

Keywords: Machine Tool, Machine Tool Module, Conversion

\section{INTRODUCTION}

Traditionally, Dedicated Manufacturing Systems (DMSs) have been designed to produce a specific part. These systems are economical when the production volumes are high and the part is produced over a long period of time. Conversely, Flexible Manufacturing Systems (FMSs) are designed to accommodate a large variety of parts even though the parts are not specified at the system design stage. For an overview of FMSs, see [1]. These systems are economical when the production volumes are low, and a large variety of parts are produced. Rapidly changing market demands have made traditional DMSs economically infeasible for many applications and have driven the use of FMSs. However, FMSs do not provide the robustness of DMSs and often have wasted resources making them uneconomical in many production situations [2]. For many applications it is believed Reconfigurable Manufacturing Systems (RMSs) that combine the advantages of DMS and FMS will provide an economical solution [3]. These systems are designed to accommodate a specific range of production requirements (i.e., product mix and volumes). Further, RMSs are characterized by customized flexibility, such that they are tailored to the current production requirements, but may be economically converted to a new set of production requirements. Thus, these systems are economical and robust since they are customized to the production requirements, their resources are minimized, and flexibility in their design allows for costeffective conversion when new production requirements arise. Indeed, reconfigurable manufacturing has been identified as one of the six grand challenges for competitive manufacturing in 2020 [4].

As production requirements for a machining system change, so will the operation requirements (i.e., features to be machined and cycle times) for an individual station. Major components that make RMSs feasible are reconfigurable machine tools (RMTs). These machine tools are cost-effective because they are custom-designed for a given range of operation requirements, and can be economically converted to meet new requirements. A conceptual design of an RMT based on the patent of Koren and Kota [5] is shown in Figure 1. As the part size and features change, the spindles can be relocated to perform the same operation in a different location or replaced with another spindle to perform a different operation. Spindles can also be added or deleted such that the resources are optimized.

Figure 1: Conceptual RMT [5].

A common type of machine tool in large manufacturing industries today is the dedicated machine tool (DMT). This type of machine tool is custom-designed for specific operation requirements and, therefore, its resources are minimized and, in turn, the machine cost is low and its performance is robust. The drawback of DMTs is that they cannot be cost-effectively converted when parts change. To address this challenge, flexible CNC machine tools have been developed and adopted in many industries. By contrast to DMTs that are designed around a specific part and therefore are inexpensive, CNCs are designed before the operation requirements are known and, thus, they often have wasted resources that make customers pay for features they do not need. Further, these machines may not be able to handle the new operation demands (e.g., spindle power, geometric accuracy).

The challenge, therefore, is to adopt the DMT approach and design a machine around a part family or a set of parts (rather than a specific part, so conversion by rapid reconfiguration of the machine is possible) and use CNC technology to drive the machine. This hybrid machine (i.e., 
the RMT), therefore, has a customized flexibility that makes it less expensive than general-purpose CNCs [6]. These novel machine tools are discussed in detail throughout this paper. One characteristic of RMTs is modularity: both in the mechanical structure and in the controller. Modular machine tools (MMTs) are being produced in industry with varying degrees of modularity. At the machine level, several companies (e.g., Lamb, Cellular Concepts, Heller, and Mazak) add and delete standard units (e.g., three perpendicular axes of motion with one cutting tool) to a line as needed. Special modular units have been developed expressly for the Russian automotive industry [7]. The concept of modular units for manufacturing equipment is also briefly discussed in the context of concurrent product and production system design [8].

Garro and Martin [9] provided an overview of the modular design of a machine tool including interactions with the environment (e.g., operators and other machines) and the determination of control modules. Zatarain et al. [10] developed a method to analyze the dynamic stiffness of a machine tool using pre-calculated component information. The notion of reconfiguration, however, extends beyond a customized-assembly of modular elements. That is, reconfigurable systems are: (i) modular in their construction and therefore can be reconfigured by swapping modules as needed, (ii) convertible: individual modules can be repositioned or re-oriented without changing the topological characteristics of the machine. This level of reconfigurability is helpful in making quick, online changes to accommodate certain changes in the product family. Thus, reconfigurable machine tools can be designed to provide different levels of reconfigurablity and the design process starts with a thorough understanding of peculiarities (geometry, processes, tolerances, cycle time, reconfiguration time) of a given family of products to be machined. Since the machine is designed around a part family, the reconfigurable machine offers customized flexibility at lower cost; that is the right kind of flexibility without any wasted-flexibility. Three methodologies are discussed in a subsequent section. A systematic methodology for the kinematic synthesis of RMTs starting from a mathematical description of the machining tasks [11], a methodology to evaluate the dynamic stiffness of RMTs [12], and one that evaluates machine dynamic errors using module information [13].

In this paper are also provided three example operation scenarios, each with different types of operation requirements, to illustrate the characteristics of RMTs and the differences between RMTs and other types of machine tools (i.e., dedicated, CNC, and modular).

\section{MANUFACTURING REQUIREMENTS}

The dominant production requirements for large machining systems are (1) mix (i.e., set of parts to be produced), and (2) volume (amount produced per unit time of each part) as well as changes in the part set and volumes over the life-time of the machining system. A variety of factors will influence the change in part sets and volumes over time including tightening government regulations, increasing (decreasing) customer demands, etc. These factors may result in slight design modifications in the parts being produced, the introduction of new parts, the phasing-out of current parts, or the increase (decrease) in the volume of each part. Similarly, the operation requirements for a machine tool consist of (1) the set of features that are produced and (2) the cycle time (i.e., time to complete the operation for one part) of each operation as well as changes in the feature sets and cycle times. Changes in the production requirements directly effect the operation requirements. A feature set may be altered (e.g., tolerance increased, hole enlarged), added or subtracted, or the required cycle time may be increased or decreased. The relationship between the production and operation requirements and the various types of machining systems and machine tools is shown in Table 1 and elaborated below.

\begin{tabular}{|l|c|c|c|c|c|c|}
\hline & DMS & DMT & FMS & CNC & RMS & RMT \\
\hline Part Mix & S & S & V & V & Fa & Fa \\
\hline Volume & F & F & C & F & C & C \\
\hline
\end{tabular}

C: changeable; F: fixed; Fa: family; S: single; V: various

Table 1: A comparison of three systems.

Dedicated machining systems (DMSs) are designed for narrowly defined production requirements (typically one part at a fixed volume) that are assumed to remain constant over the life-time of the machining system. These systems are comprised of dedicated machine tools (DMTs), each of which is custom-designed to machine a specific set of features (often a single feature) at a constant cycle time. Therefore, while DMSs are tailored to their production requirements and, thus, are robust and inexpensive, they cannot cost-effectively accommodate changes in production requirements. Similarly, DMTs are tailored to specific operation requirements and, in general, cannot cost-effectively accommodate changes in the operation requirements. If the volume sharply increases, the DMS may not be able to accommodate the increase and another system will need to be built or the sale opportunity will be lost. A slight design change to the part may trigger the need to modify one or more DMTs. As these machine tools are not designed such that they may be cost-effectively converted, the redesign and ramp-up of a modified (or entirely new) DMT will often be too costly. Further, the introduction of an entirely new part will require the design of an entirely new machining system.

Flexible manufacturing systems (FMSs) are designed for loosely defined production requirements that are assumed to significantly change in an unknown manner over time. Since the production requirements are not well defined, FMSs often contain excessive capability, and the customer pays for unneeded capabilities. Generalpurpose Computer Numerical Control (CNC) machine tools are the building blocks of FMSs. Loosely defined production requirements at the system level translate into loosely defined operation requirements at the machine level. As a result, CNCs often contain excessive functionality (e.g., a five-axis CNC may use only two axes in a given operation, or only 6 tools of a 24-tool magazine may be utilized). Further, since CNCs are typically not designed for a specific set of operations, extensive testing must be performed to ensure the quality requirements will be met in the entire machine envelope. Increases in volume typically require additional CNCs.

Reconfigurable Machining Systems (RMSs), which are designed for a specific range of production requirements, cost-effectively combine the most attractive features of DMSs and FMSs: robust performance and the ability to accommodate new production requirements [14] Reconfigurable Machining Systems contain a combination of DMTs and CNCs, and incorporate, where appropriate, RMTs that are designed to produce specific sets of features for specific ranges of cycle times. Some operation requirements will be constant over the life-time of the machining system and, thus, DMTs will be the appropriate choice for these operation requirements. Some operation requirements will change dramatically in an unknown manner over the life-time of the machining system and, thus, CNCs will be the appropriate choice for these operation requirements. Since RMTs are designed for specific sets of features and ranges of cycle times, RMTs 
are tailored to the initial operation requirements and, when operation requirements change, RMTs may be costeffectively converted such that they are customized for the new requirements.

\section{RMT CONTROL REQUIREMENTS}

The control components (hardware and software) of DMTs are customized for the DMT's requirements and, thus, do not contain unnecessary complexity and are correspondingly robust. However, these components cannot be cost-effectively upgraded. Typical CNC controllers posses comprehensive architectures to provide processing flexibility; however, not all of the built-in functionality may be used. Thus, unnecessary costs are incurred due to software development, installation, and especially in maintenance and diagnostics. Further, similar to its mechanical components, CNC controllers posses control hardware resources (e.g., data acquisition boards, motor drives) that are often underutilized. For both DMTs and CNCs, the control components are not modular and, thus, are not scalable nor upgradable and new technology (e.g., advanced geometric compensation) cannot be costeffectively integrated.

Controllers for RMTs must be based on the concept of open-architecture [15-17]. In open-architecture control, the software architecture is modular and, thus, hardware components (e.g., encoder) and software components (e.g., axis control logic) can be easily added or removed, and the controller can be cost-effectively reconfigured. The RMT controller modularity allows the controller to be customized to its current operation requirements and, thus, be robust and reliable, while maintaining the ability to be reconfigured when requirements change or new technology becomes available.

Reconfiguration requirements introduce several new challenges for RMT controllers. The first challenge is the reconfiguration of the controller architecture that is required when the physical machine tool is reconfigured or new technology is integrated. Unlike DMT or CNC controller architectures, the RMT controller architecture is dynamic. For example, the addition of a linear axis to a one-axis RMT may require the integration of an interpolator software module. Another challenge is the control of RMTs with multiple tools working independently (as in Figure 1) and RMTs with axes in non-orthogonal configurations (see the Second Example below). Strategies have been developed for the interpolation and control of RMTs with axes in non-orthogonal configurations. Another challenge is the integration of heterogeneous software and hardware components (e.g., fieldbus protocols, control signals, electrical contacts) that are developed by different vendors at different times. This will require standard software and electrical interfaces or the development of special components that interface custom devices to standard interfaces.

To handle the challenge of cost-effective reconfiguration of RMT controllers, work on a software tool known as a control configurator is being developed and is currently being applied to a prototype RMT (see the First Example). The controller (Figure 2) is composed of a configuration tool, a simulation tool, and a common HMl. The configuration tool is used to reconfigure the software whenever the prototype RMT structure is reconfigured (i.e., a linear axis is added or deleted). The tool allows the user to reconfigure the controller via a graphical user interface and generate the required software for the PC-based open architecture controller. The real-time simulation tool simulates the dynamics and discrete events of the electromechanical components and the machining process. This simulation is connected to the actual machine tool controller; thus, the user is able to evaluate and debug the controller without operating the real machine whenever the controller is reconfigured.

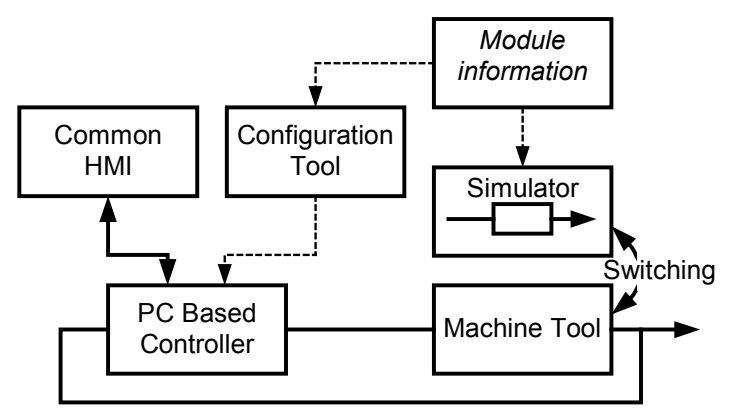

Figure 2: Structure of prototype RMT controller.

\section{RMT MECHANICAL REQUIREMENTS}

For a machine tool to meet the productivity and quality demands of an operation, it must fulfill a variety of requirements including the ability to produce the specified motions and satisfy the part tolerance specifications. To produce the required motions, the machine tool's kinematic capabilities must be examined. To meet the part tolerance requirements, the mechanical sources of error (e.g., component geometric error, assembly errors, thermal deformation) must be examined. The following discusses two CAD/CAM tools that have been developed for RMT mechanical design.

Kinematic Viability. One requirement in the design of a machine tool is kinematic viability (i.e., the machine tool must be able to perform the motions required to produce the feature set). For a DMT, the minimum required degrees of freedom (DOF) is designed given the feature set to be machined. The kinematic configuration, therefore, is limited. For a CNC, the entire machine tool, (typically 3-5 axes), is designed before the required set of operations is known. Therefore, CNCs typically have the required DOF for any operation; however, for many operations, the CNC possesses extra DOF creating wasted resources and unnecessary complexity.

Similar to a DMT, the DOF of an RMT is designed after the operation set has been determined; however, the change in operation requirements, in addition to the initial operation set, must also be considered. As the operation requirements change, the DOF requirements may also change and, thus, the RMT will need to be mechanically modular to adapt to these changes. However, RMTs will not be general-purpose modular machine tools; instead, they will be designed with the minimal amount of required modularity. This amount will be dictated by the range of operations that are required of the machine tool and the frequency of the change in these operations.

A methodology (Figure 3) has been developed to determine the RMT kinematic requirements automatically [11]. The machining operation is transformed into a task matrix (i.e., a homogeneous transformation matrix [HTM]) that contains the necessary motion requirements for the machine tool. The functional requirements of the machining operation are used to generate graph representations of candidate machine tools. A graph gives the overall topology of the machine tool and structural and kinematic functions are assigned to various portions of the graph. A library of machine tool modules (e.g., spindles, slides) containing structural and kinematic information for each module via HTMs, as well as connectivity information is examined. Modules are assigned to various portions of the graph. The product of their HTMs is compared to the task matrix. If these matrices are equal, then the machine tool is kinematically viable. In this manner, all possible configurations can be determined. The viable 
configurations will be further reduced by other criteria (e.g. DOF, static and dynamic stiffness, thermal growth characteristics). This methodology also determines which modules must be added or deleted for each part in the part family. A CAD/CAM machine tool design package called PREMADE has been developed to assist the machine tool designer in implementing these algorithms.

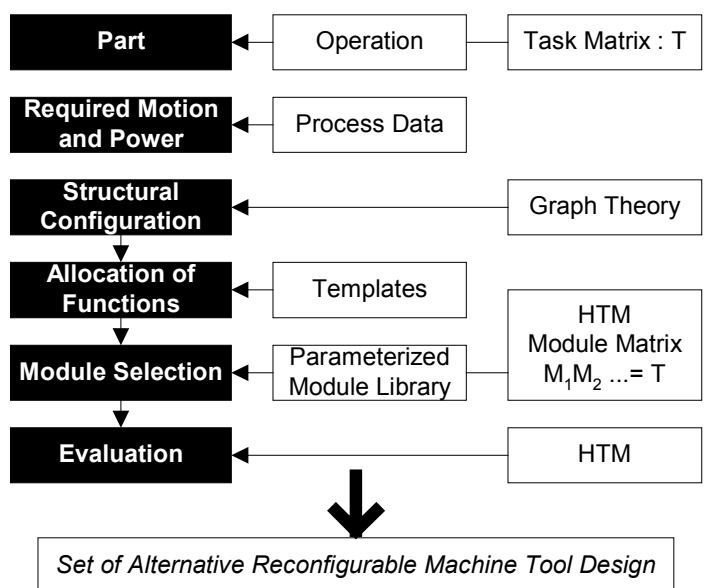

Figure 3: RMT kinematic design methodology.

Structural Stiffness. One of the most critical design criteria for machine tools is structural stiffness. Static deflections cause geometric errors, and chatter may result if the dynamic structural characteristics are not properly designed. Based upon the designer's experience, DMTs are designed to have the required stiffness characteristics for a specific operation. Conversely, CNCs are designed before the operation requirements are known; thus, extensive testing must be performed to ensure excessive deflections and chatter will not occur when a new operation is introduced.

Similar to DMTs, RMTs are designed such that the structural stiffness requirements are ensured; however, RMTs have additional requirements. First, structural stiffness must be guaranteed for all configurations the RMT may take, and for all operations that may be performed. Also, RMT joints, in general, will be designed such that mechanical modules may be cost-effectively rearranged and; thus, the joints cannot be treated as rigid (i.e., their compliance and damping are significant). Therefore, joint stiffness will dramatically affect the overall machine tool structural stiffness and must be carefully taken into account during the analysis stage.

A systematic methodology is being developed to evaluate the structural stiffness of RMTs [12]. The critical process parameters (e.g., cutting force magnitude and frequency content) are identified for the range of possible operations. For each design candidate, the joint parameters are determined assuming the joint model, or its describing function, is available. A substructuring method called nonlinear receptance coupling is used and the structural stiffness of each candidate is determined. The candidates are then evaluated using criteria such as static stiffness, fundamental frequency, minimum and mean dynamic stiffness within the frequency of interest, and coefficient of merit which evaluates performance with respect to chatter.

An alternative to structural stiffness design is the use of vibration isolation systems (active, passive, or hybrid). Various vibration isolation strategies for RMTs were proposed by Yigit and Ulsoy [18]. Nevertheless, careful structural stiffness design coupled with vibration isolation, where needed, will generally be preferred.

Geometric Accuracy. Another machine tool design criterion is geometric accuracy. Machine tool geometric errors create part geometry errors and, thus, dramatically affect part quality. Sources of machine tool geometric errors include tolerance errors in subcomponents, motion errors, static deflections due to weight and machining forces, thermal deformations, spindle runout, and assembly errors. A DMT is designed, based on the designer's experience, so the machine tool geometric errors are such that the part tolerance specifications will be met. Given the complex nature of machine tool geometric errors, extensive testing is often required to ensure part tolerance requirements are met for high precision applications. For a CNC, the geometric errors are minimized given the cost constraint for the machine tool. Since the CNC is designed before the part is selected, extensive testing is often required to ensure part tolerance requirements are met.

Similar to a DMT, the RMT mechanical structure is designed such that the machine tool's geometric errors will not compromise part quality; however, two additional considerations must be taken into account. First, since RMTs are designed for a range of operation requirements, the most limiting part tolerance requirements will dictate the geometric error requirements of the machine tool. Second, the structure of an RMT may need to be reconfigured; therefore, for some applications, RMTs will require mechanical adapters that allow for the quick and accurate addition or deletion of mechanical modules. An overview of the repeatability properties of a variety of viable adapters is given in [19].

A systematic methodology is being developed to analyze the accuracy of RMTs [13]. An overview of the methodology is shown in Figure 4. Each module will have geometric, and possibly motion, related errors. The errors of existing modules may be represented deterministically if measurements have been performed. Otherwise, the errors are represented in a statistical manner. Assembly errors are also represented statistically. The geometric errors of each machine tool component and the assembly errors are mathematically described by HTMs, similar to the kinematic representation. Monte Carlo simulations are used to analyze the distribution of the tool position errors given the statistical distribution of component and assembly errors. The data is post processed and criteria (e.g., reliability, robustness) are being developed to systematically compare various machine tools. This methodology will be extended to the analysis of static errors resulting from machining forces and thermal deformation, and analysis modules will be written to integrate with the PREMADE software.

\begin{tabular}{|c|c|}
\hline $\begin{array}{l}\text { (1) Statistical Id } \\
\text { New Modules, } \\
\text { Machining For }\end{array}$ & $\begin{array}{l}\text { ication of Error Source } \\
\text { sed Modules, Adapters, } \\
\text { emperature }\end{array}$ \\
\hline$\downarrow$ & \\
\hline $\begin{array}{l}\text { (2) Error Model } \\
\text { (HTM) }\end{array}$ & $\begin{array}{l}\text { Standardized Module Library } \\
\text { Kinematic information, }\end{array}$ \\
\hline$\checkmark$ & Tolerance, Adapter assembly \\
\hline (3) Simulation & error, Stiffness \\
\hline$\checkmark$ & \\
\hline $\begin{array}{l}\text { Results } \\
\text { Final Error (Sta } \\
\text { Error Signature }\end{array}$ & $\begin{array}{l}\text { al Prediction) } \\
\text { ect of each source) }\end{array}$ \\
\hline$\downarrow$ & \\
\hline $\begin{array}{l}\text { (4) Statistical Id } \\
\text { Performance E } \\
\text { Decision Makir }\end{array}$ & $\begin{array}{l}\text { ication of Error Source } \\
\text { ation, Tolerance Allocation, }\end{array}$ \\
\hline
\end{tabular}

Figure 4: RMT error evaluation methodology. 


\section{FIRST EXAMPLE - PART CHANGE}

Examples of machine tool reconfiguration are presented in the following three sections.

The first example considers the design of an RMT for the drilling process of precision cam tower holes. The part family consists of two automotive cylinder heads: a V8 single overhead cam and a V6 duel overhead cam (Figure 5). A machining line is to be designed to initially produce V8 heads. However, the line must have the capability to produce V6 heads when market demands changes; however, the line will produce only one head at any given time. As seen in Figure 5, each cam cap is located on its respective cam tower via two dowel pins; therefore, the cam tower holes which hold the dowel pins must be machined to tight tolerances. The V8 head has two cam caps and requires four precision holes that are colinear. The V6 head, however, has eight cam caps and sixteen precision holes that are not colinear. A machine tool will require at least two axes of motion (one to locate and one to feed) to produce the V8 head and at least three axes of motion (two to locate and one to feed) to produce the V6 head.

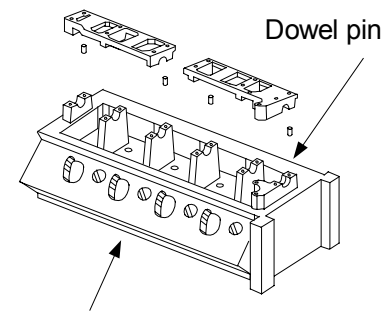

Milling surface $45^{\circ}$

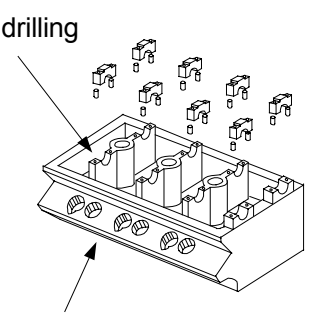

Milling surface $30^{\circ}$
Figure 5: Part family - two automotive cylinder heads: V8 (left) and V6 (right).

A large number of RMTs can be realized for these operation requirements; indeed, the PREMADE software discussed above determined scores of solutions given a modest module library. In this section a prototype RMT that was designed for these operation requirements [6] will be described. The prototype RMT is shown in Figure 6. For the V8 head, the two-axis configuration of the RMT is employed: the horizontal linear axis positions the selffeeding spindle. To machine the V6 head, the three-axis configuration is employed. The prototype RMT is designed such that it may be easily converted between the two and three-axis configurations. This is accomplished by means of a standard interface on the saddle of both linear axes. This interface uses Ball Locks that allow mechanical components to be joined quickly (a screw turn locks the balls into the receiver bushing) and accurately (the primary Ball Locks and their associated bushings are precision machined). The spindle may be joined to the saddle of either axis and the vertical axis may be joined to the horizontal axis as well. When the prototype RMT is reconfigured mechanically, the controller must be modified. This cannot be accomplished using a PLC or a CNC controller; therefore, an open controller was utilized (Figure 2 ). This controller was modified such that one axis controller may be turned off or on via a screen command. The modular structure of the prototype RMT (in both hardware and software) allows it to be converted in a costeffective manner when the requirements change and provide a customized solution for both operations.
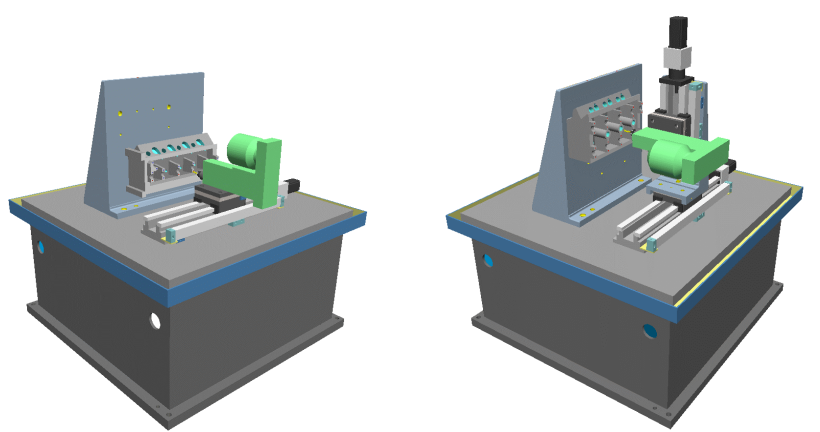

Figure 6: Prototype RMT: two-axis (left) and three-axis (right) [6].

\section{SECOND EXAMPLE - FEATURE CHANGE}

The part family and production requirements in the second example are the same as in the first example (Figure 5). However, this example considers the design of an RMT for finish milling of the cylinder head inclined surfaces. The tooling, tolerances, process parameters, etc. are the same for both inclined surfaces; however, the surfaces of the V6 and V8 cylinder heads have different angles with respect to horizontal: $30^{\circ}$ and $45^{\circ}$, respectively. If the cylinder heads were being produced on a DMS, a DMT would be customdesigned with one axis for the V8 cylinder head that is $30^{\circ}$ from horizontal. This would be the most economical solution if the V6 was never introduced. However, a new custom-designed DMT with one axis at a $45^{\circ}$ angle would need to be built and tested for the V6 inclined surface finish milling operation if the V6 was introduced at a later date. In an FMS, a CNC station would require four or five axes (depending on the fixture and process plan) whereas only 3 motions are needed thus, there would be one or two wasted axes of motion. However, the introduction of the V6 cylinder head would just require a change in the part program.

In an RMS environment, an RMT would be used where the spindle unit could be placed on the machine tool structure in two different locations such that the spindle unit was at either a $30^{\circ}$ or a $45^{\circ}$ angle with respect to the horizontal. A new type of RMT, called the Arch-Type RMT (AT-RMT) is being developed [20] for these types of operations. As seen in Figure 7, the AT-RMT has two predetermined spindle unit locations. It is a non-orthogonal 3-axis machine designed around parts with inclined surfaces. The spindle angle and position could be adjusted before the operation either by using an actuator on the arch axis or manually.
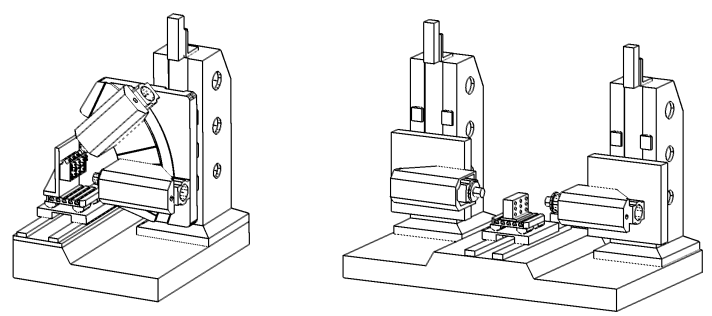

Figure 7: AT-RMT (left) and Parallel RMT (right).

\section{THIRD EXAMPLE - CYCLE TIME CHANGE}

The part family for the third example is a simple prismatic part and the operation that is considered is drilling an identical pattern of holes on two sides opposite of one another. Initially, the cycle time is such that a single spindle may be used: one side is machined, the part is indexed $180^{\circ}$, and the second side is machined. Further, it is assumed that the final product is produced in a system containing several stations and that this particular 
operation has the longest cycle time. In this example, the affect of increased product demand and, hence, decreased cycle time, is explored.

Since the operation that is considered is the bottleneck operation, if a DMS was utilized, the only way to increase the capacity of the system would be to build another line. If the increase in product demand were less than double, this would result in an underutilized system. Further, if demand returned to normal in the future, an entire machining line would remain idle. If an FMS was utilized, and additional station would be required, which would increase the cost.

In an RMS, each station would be designed such that it may be cost-effectively reconfigured, if required, to meet the increase in demand. For the operation being considered, one solution is to add another spindle opposite to the first, remove the indexing table, and simultaneously machine both sides (see Figure 7). This solution is based on the methodology of using multiple independent spindles for RMTs [5]. More than half of the cycle time will be saved with this solution since the part will no longer need to be indexed.

\section{SUMMARY AND CONCLUSIONS}

This paper provided a detailed overview of a new paradigm for machine tools - reconfigurable machine tools. The effect of production and operation requirements on the design of RMTs was explored. The mechanical and control requirements of RMTs were given and systematic design tools that have been recently developed were reviewed. Three examples, illustrating various changes in operation requirements, were provided and RMTs were compared to dedicated machine tools, modular machine tools, and flexible CNCs.

Reconfigurable machine tools provide a viable solution for manufacturing situations where operation requirements change within prescribed bounds over the life-time of the machine tool. Like DMTs, RMTs are customized to their current operation requirements and, thus, are robust; however, RMTs are also designed such that they may be cost-effectively converted when operation requirements change. Further, unlike CNCs that can accommodate a wide variety of operation requirements, RMTs are designed for a specific range of operation requirements and, thus, do not have wasted resources and functionality. Reconfigurable machine tools are also capable of costeffectively incorporating new technology, thereby extending the use of the machine tool. To provide an economical solution, systematic design tools will be needed to quickly analyze a variety of RMTs that must perform over a range of operation requirements. Such tools will include kinematic synthesis, structural stiffness and error analyses, controller configuration, etc. The examples provided in this paper have also illustrated the dramatic effect that production and operation requirements have on the design of RMTs and on the different types of RMT conversions (e.g., adding an additional axis, reorienting an axis, integrating new control and diagnostic algorithms) that are needed.

\section{ACKNOWLEDGMENTS}

The authors gratefully acknowledge the financial support of the Engineering Research Center for Reconfigurable Machining Systems (NSF Grant EEC95-92125) at the University of Michigan and the valuable input from the Center's industrial sponsors.

\section{REFERENCES}

[1] Sethi, A.K. and Sethi, S.P., 1990, Flexibility in Manufacturing: A Survey, International Journal of Flexible Manufacturing Systems, Vol. 2, pp. 289-328.
[2] Mehrabi, M.G. and Ulsoy, A.G., 1997, State-of-theArt in Reconfigurable Machining Systems, ERC/RMS Technical Report, University of Michigan, Ann Arbor, Michigan.

[3] Koren, Y., Heisel, U., Jovane, F., Moriwaki, T., Pritschow, G., Ulsoy, G., and Van Brussel, H., 1999, Reconfigurable Manufacturing Systems, Annals of the CIRP, Vol. 48/2, pp. 527-540.

[4] Bollinger, J. et al., 1998, Visionary Manufacturing Challenges for 2020, National Research Council Report, National Academy Press, Washington, D.C.

[5] Koren, Y. and Kota, S., 1999, Reconfigurable Machine Tools, U.S. Patent 5,943,750.

[6] Landers, R.G., 2000, A New Paradigm in Machine Tools: Reconfigurable Machine Tools, Japan-USA Symposium on Flexible Automation, Ann Arbor, Michigan, July 23-26.

[7] Genin, V.B. and Kozlov, A.I., 1990, New Modules Offer Enhanced Flexibility for Automatic Lines, Soviet Engineering Research, Vol. 61, No. 2, pp. 14-17.

[8] Rogers, G.G. and Bottaci, L., 1997, Modular Production Systems: a New Manufacturing Paradigm, Journal of Intelligent Manufacturing, Vol. 8, pp. 147156.

[9] Garro, O. and Martin, P., 1993, Towards New Architectures of Machine Tools, International Journal of Production Research, Vol. 31, No. 10, pp. 24032414.

[10] Zatarain, M., Lejardi, E., and Egana, F., 1998 , Modular Synthesis of Machine Tools, Annals of the CIRP, Vol. 47/1, pp. 333-336.

[11] Moon, Y-M. and Kota, S., 1999, A Methodology for Automated Design of Reconfigurable Machine Tools, Proceedings of the $32^{\text {nd }}$ CIRP International Seminar on Manufacturing Systems, Leuven, Belgium, May, pp. 297-303.

[12] Yigit, A.S. and Ulsoy, A.G., 2001, Dynamic Stiffness Evaluation for Reconfigurable Machine Tools Including Weakly Nonlinear Joint Characteristics, Proc. IME, Part B: Journal of Engineering Manufacture (submitted).

[13] Moon, S-K., Landers, R.G., and Kota, S., 2000, Error Analysis in Reconfigurable Machine Tool Design, ERC/RMS Technical Report, University of Michigan, Ann Arbor, Michigan.

[14] Koren, Y., Hu, J., and Weber, T., 1998, Impact of Manufacturing System Configuration on Performance, Annals of the CIRP, Vol. 47/1, pp. 689698.

[15] Koren, Y., Jovane, F., and Pritschow, G. (Eds.), 1998, Open Architecture Control Systems: Summary of Global Activity, ITIA Series, Vol. 2.

[16] Pritschow, G., Daniel, C.H., Jurghans, G., Sperling, W., 1993, Open Systems Controllers - A Challenge for the Future of the Machine Tool Industry, Annals of the CIRP, Vol. 42/1, pp. 449-452.

[17] Pritschow, G. et al., 2001, Open Architecture Controller, Annals of the CIRP, Vol. 50/2

[18] Yigit, A.S. and Ulsoy, A.G., 2000, Design of Vibration Isolation Systems for Reconfigurable Precision Equipment, Japan-USA Symposium on Flexible Automation, Ann Arbor, MI, July 23-26.

[19] Li, H., Landers, R.G., and Kota, S., 2000, A Review of Feasible Joining Methods for Reconfigurable Machine Tool Components, Japan-USA Symposium on Flexible Automation, Ann Arbor, MI, July 23-26.

[20] Katz, R. and Chung, H., 2000, Design of an Experimental Reconfigurable Machine Tool, JapanUSA Symposium on Flexible Automation, Ann Arbor, MI, July 23-26. 